\title{
Antibody response to multiple antigens of SARS-CoV-2 in patients with diabetes: an observational cohort study
}

\author{
Vito Lampasona ${ }^{1}$ (D) Massimiliano Secchi ${ }^{1}$ (D) Marina Scavini $^{1}$ (D) Elena Bazzigaluppi ${ }^{1}$ (D) Cristina Brigatti $^{1} \cdot$ \\ Ilaria Marzinotto ${ }^{1}$ (I) $\cdot$ Alberto Davalli ${ }^{1} \cdot$ Amelia Caretto $^{1}$ (D) $\cdot$ Andrea Laurenzi ${ }^{1} \cdot$ Sabina Martinenghi ${ }^{1}$ (D) \\ Chiara Molinari $^{1}$ (D) $\cdot$ Giordano Vitali $^{1} \cdot$ Luigi Di Filippo $^{1}$ - Alessia Mercalli ${ }^{1}$ (D) $\cdot$ Raffaella Melzi $^{1}$ (D) $\cdot$ Cristina Tresoldi $^{2}$. \\ Patrizia Rovere-Querini ${ }^{3,4}$ (D) $\cdot$ Giovanni Landoni ${ }^{4,5}$ (D) $\cdot$ Fabio Ciceri ${ }^{4,6}$ (D) $\cdot$ Emanuele Bosi $^{1,4}$ (D) Lorenzo Piemonti $^{1,4}$ (D)
}

Received: 30 July 2020 / Accepted: 9 September 2020 / Published online: 8 October 2020

(C) Springer-Verlag GmbH Germany, part of Springer Nature 2020

\begin{abstract}
Aims/hypothesis The aim of the study was to characterise the humoral response against severe acute respiratory syndrome coronavirus 2 (SARS-CoV-2) in patients with diabetes. Demonstrating the ability to mount an appropriate antibody response in the presence of hyperglycaemia is relevant for the comprehension of mechanisms related to the observed worse clinical outcome of coronavirus disease 2019 (COVID-19) pneumonia in patients with diabetes and for the development of any future vaccination campaign to prevent SARS-CoV-2 infection.

Methods Using a highly specific and sensitive measurement of antibodies by fluid-phase luciferase immunoprecipitation assays, we characterised the IgG, IgM and IgA response against multiple antigens of SARS-CoV-2 in a cohort of 509 patients with documented diagnosis of COVID-19, prospectively followed at our institution. We analysed clinical outcomes and antibody titres according to the presence of hyperglycaemia, i.e., either diagnosed or undiagnosed diabetes, at the time of, or during, hospitalisation.

Results Among patients with confirmed COVID-19, 139 (27.3\%) had diabetes: 90 (17.7\%) had diabetes diagnosed prior to the hospital admission (comorbid diabetes) while $49(9.6 \%)$ had diabetes diagnosed at the time of admission (newly diagnosed). Diabetes was associated with increased levels of inflammatory biomarkers and hypercoagulopathy, as well as leucocytosis and neutrophilia. Diabetes was independently associated with risk of death (HR 2.32 [95\% CI 1.44, 3.75], $p=0.001$ ), even after adjustment for age, sex and other relevant comorbidities. Moreover, a strong association between higher glucose levels and risk of death was documented irrespective of diabetes diagnosis (HR $1.14 \times 1.1 \mathrm{mmol} / 1[95 \% \mathrm{CI} 1.08,1.21], p<0.001)$. The humoral response against SARS-CoV-2 in patients with diabetes was present and superimposable, as for timing and antibody titres, to that of non-diabetic patients, with marginal differences, and was not influenced by glucose levels. Of the measured antibody responses, positivity for IgG against the SARS-CoV-2 spike receptor-binding domain (RBD) was predictive of survival rate, both in the presence or absence of diabetes.

Conclusions/interpretation The observed increased severity and mortality risk of COVID-19 pneumonia in patients with hyperglycaemia was not the result of an impaired humoral response against SARS-CoV-2. RBD IgG positivity was associated
\end{abstract}

Electronic supplementary material The online version of this article (https://doi.org/10.1007/s00125-020-05284-4) contains peer-reviewed but unedited supplementary material, which is available to authorised users.

Lorenzo Piemonti

piemonti.lorenzo@hsr.it

$1 \quad$ San Raffaele Diabetes Research Institute, IRCCS Ospedale San Raffaele, Milan, Italy

2 Molecular Hematology Unit, IRCCS Ospedale San Raffaele, Milan, Italy

3 Department of Immunology, Transplantation and Infectious Diseases, IRCCS Ospedale San Raffaele, Milan, Italy
4 School of Medicine and Surgery, Università Vita-Salute San Raffaele, Milan, Italy

5 Department of Anesthesia and Intensive Care, IRCCS Ospedale San Raffaele, Milan, Italy

6 Hematology and Bone Marrow Transplantation Unit, IRCCS Ospedale San Raffaele, Milan, Italy 


\section{Research in context}

\section{What is already known about this subject?}

- Diabetes has been associated with an excess risk of severe COVID-19 pneumonia

- Individuals with diabetes have a consistently decreased humoral response to the hepatitis B vaccine, while less consistent results were noted for influenza and varicella zoster vaccines

- The immunological humoral response against SARS-CoV-2 in patients with hyperglycaemia has not yet been studied

What is the key question?

- Is the worse clinical outcome of COVID-19 in patients with diabetes associated with a quantitative or qualitative defect of the antibody response against the SARS-CoV-2 virus?

\section{What are the new findings?}

- The antibody response against multiple SARS-CoV-2 antigens in patients with diabetes is superimposable, as for timing, titres and classes, to that of non-diabetic patients and is not influenced by glucose levels

- Blood glucose levels are strongly associated with an increased mortality risk for COVID-19 pneumonia, even in patients without diabetes

- Positivity for IgG against the SARS-CoV-2 spike receptor-binding domain is predictive of survival rate both in the presence and absence of diabetes

How might this impact on clinical practice in the foreseeable future?

- Hyperglycaemia does not impair the antibody response against SARS-CoV-2, allowing for optimism regarding the efficacy of future vaccines against SARS-CoV-2 in patients with diabetes

with a remarkable protective effect, allowing for a cautious optimism about the efficacy of future vaccines against SARs-COV-2 in people with diabetes.

Keywords Antibodies · COVID-19 · Diabetes · Human · Humoral response · Receptor-binding domain · SARS-CoV-2 . Survival rate

Abbreviations
AU
COVID-19
CRP
eCRF
FPG
ICU
IRCCS
LDH
LIPS
NP
RBD

S1, S2

SARS-CoV-2
Arbitrary units

Coronavirus disease 2019

C-reactive protein

Electronic case record form

Fasting plasma glucose

Intensive care unit

Istituto di Ricovero e Cura a

Carattere Scientifico

Lactate dehydrogenase

Luciferase immunoprecipitation system

Nucleocapsid protein

Receptor-binding domain

(of SARS-CoV-2 spike protein)

Subunits of SARS-CoV-2 spike protein

Severe acute respiratory syndrome

coronavirus 2

\section{Introduction}

Severe acute respiratory syndrome coronavirus-2 (SARSCoV-2), the causative agent of coronavirus disease-2019 (COVID-19) pneumonia, has spread rapidly worldwide since its detection in China in 2019 [1]. The symptoms of COVID19 pneumonia range from very mild to severe [2]. Since the first reports, diabetes has been associated with an excess risk of severe/critical illness [3-7], and a recent meta-analysis of 89 observational studies showed that patients with COVID-19 pneumonia and established diabetes had an approximate twofold increased risk of requiring admission to an intensive care unit (ICU) and a threefold increased risk of in-hospital mortality [8]. Similar excess risk among patients with diabetes has been reported for the two earlier severe coronavirus infections, i.e. severe acute respiratory syndrome (SARS) [9] and Middle East respiratory syndrome (MERS) [10, 11]. Several 
putative pathophysiological mechanisms [12] have been proposed for the observed worse clinical outcome of COVID-19 pneumonia in patients with diabetes: (1) higher affinity cellular binding and more efficient virus entry [13-16]; (2) more common use of agents able to modulate ACE2 expression (glucose-lowering agents such as glucagon-like peptide-1 agonists and thiazolidinediones, antihypertensive drugs such as ACE inhibitors, and statins) [17-19]; (3) decreased viral clearance [20]; (4) increased susceptibility to hyperinflammation and cytokine storm syndrome [21]; (5) presence of comorbidities, such as hypertension and/or cardiovascular disease and/or obesity, as well as older age [3-7]; and (6) impaired immunological function [22]. However, many of these proposed mechanisms are predominantly untested hypotheses or theories, based on observational data. Although several defects in immunity have been associated with hyperglycaemia/insulin resistance, and international guidelines recommend additional vaccinations for people with diabetes, the complexity of the humoral response against SARS-CoV-2 in patients with hyperglycaemia has not yet been studied. Demonstrating the ability to mount an appropriate antibody response in the presence of hyperglycaemia, associated with either diagnosed or undiagnosed diabetes, is extremely relevant for future vaccination campaigns to prevent SARS-CoV-2 infection and COVID-19 pneumonia in individuals with diabetes. Using highly specific and sensitive measurements of antibodies by fluid-phase luciferase immunoprecipitation assays, we characterised the $\operatorname{IgG}$, IgM and $\operatorname{IgA}$ response against multiple antigens of SARS-CoV-2 and analysed them according to hyperglycaemia, i.e., known or newly diagnosed diabetes. Our study cohort consisted of 509 patients with confirmed COVID-19 pneumonia admitted to the Emergency or Clinical departments at the San Raffaele Hospital in Milan between 25 February and 19 April 2020 and prospectively followed for clinical outcome.

\section{Methods}

Study population and data sources The study population consisted of adult patients ( $\geq 18$ years) with suspected COVID-19 pneumonia admitted between 25 February and 19 April 2020 to the Emergency or Clinical departments of the Istituto di Ricovero e Cura a Carattere Scientifico (IRCCS) San Raffaele Hospital and for whom a serum sample was stored in our institution biobank. This series of patients is part of the COVID-19 institutional clinical-biological cohort, assessing patients with COVID-19 at our institution, a 1350 bed tertiary care hospital in Milan, Italy (COVID-BioB; ClinicalTrials.gov Identifier: NCT04318366). The reorganisation of the hospital to face the COVID-19 outbreak and the hospital guidelines for the management of respiratory failure have been recently reported [23, 24]. The study was approved by the Institutional Review Board (IRB; protocol number 34/int/2020). Informed consent was obtained according to IRB guidelines. A confirmed infection case was defined as a SARS-CoV-2-positive RT-PCR test from a nasal/throat swab, and/or signs, symptoms and radiological findings suggestive of COVID-19 pneumonia. Data were collected from medical chart review or directly by patient interview, and entered in a dedicated electronic case record form (eCRF) specifically developed on site for the COVID-BioB study. Before analysis, eCRF data were crosschecked in a blinded fashion with medical charts and verified by data managers and clinicians for accuracy. Routine blood tests included complete blood count with differential, renal and liver function tests, C-reactive protein (CRP), lactate dehydrogenase (LDH), serum ferritin, D-dimer and IL-6.

Definition of diabetes Study participants were defined as having: (1) comorbid diabetes if they had a documented diagnosis of diabetes before the hospital admission for COVID-19 pneumonia (fasting plasma glucose $[\mathrm{FPG}] \geq 7.0 \mathrm{mmol} / \mathrm{l}$ or $\mathrm{HbA}_{1 \mathrm{c}} \geq 48 \mathrm{mmol} / \mathrm{mol}$ [6.5\%], or they were taking glucoselowering medications); (2) newly diagnosed diabetes if patients without a diagnosis of diabetes had a mean FPG $\geq 7.0 \mathrm{mmol} / \mathrm{l}$ during the hospitalisation for COVID-19 pneumonia. If not specified, the term 'diabetes' includes either comorbid or newly diagnosed diabetes. We computed mean FPG and glucose variability (SD) from all fasting laboratory glucose values measured during hospitalisation. Patients with diabetes were classified as having pancreatogenic and metasteroid diabetes, respectively, when their diabetes was with reasonable certainty secondary to their exocrine pancreatic disease or steroid use; type 1 diabetes when diabetes at onset was associated with evidence of islet autoimmunity; type 2 diabetes when patients did not fall in any of the previous classifications.

Anti-SARS-CoV-2 antibody determination To investigate the presence of anti-SARS-CoV-2 antibodies in patients with COVID-19 pneumonia, we developed a novel assay based on the luciferase immunoprecipitation system (LIPS) format [25]. LIPS is a derivative of the radio-binding assay in which serum antibodies are used to immunoprecipitate radiolabelled antigens in the liquid phase. In LIPS, the use of radiolabelled tracers is superseded in favour of chimeric recombinant antigens tagged with a luciferase reporter. The antigens used in this study were constructed in our lab [25]: sNLuc-SARS$\mathrm{CoV}-2$ beta coronavirus $\mathrm{S} 1$ receptor-binding domain (RBD) aa 319-541; sNLuc-SARS-CoV-2 beta coronavirus S1+S2 spike protein; and NLuc-SARS-CoV-2 beta coronavirus nucleocapsid protein (NP). For the LIPS assay, the antigen of interest was diluted in $20 \mathrm{mmol} / \mathrm{l}$ Tris buffer, $150 \mathrm{mmol} / 1$ $\mathrm{NaCl}, 0.5 \%$ Tween-20, pH 7.4 (TBST) buffer and adjusted to 
achieve a luciferase activity corresponding to a final concentration of $4 \times 106$ light units (LU)/25 $\mu$ l. For antibody measurement, $1 \mu \mathrm{l}$ (for IgG or IgA measurements) or $5 \mu \mathrm{l}$ (for IgM measurement) of patient serum was then seeded into the well of a 96-deep-well plate (Beckman Coulter, Brea, CA, USA), together with $25 \mu \mathrm{l}$ of the diluted antigen preparation, and incubated for $2 \mathrm{~h}$ at room temperature. For IgG antibody measurement, immunocomplexes were then captured with $5 \mu \mathrm{l}$ of blocked rProtein A [26] (GE Healthcare Europe, Freiburg, Germany) for $1 \mathrm{~h}$ at $4^{\circ} \mathrm{C}$ with shaking. For IgM and $\operatorname{IgA}$ antibody measurements, rProtein $\mathrm{A}$ was replaced with $5 \mu$ l of goat anti-human IgM- or anti-human IgA agarose (Merck Life Sciences, Milan, Italy), respectively. After washing, the resin pellets were transferred to an OptiPlate 96-well plate (PerkinElmer, Waltham, MA, USA) and upon the addition of $40 \mu \mathrm{l} /$ well of Nano-Glo substrate (Promega, Madison, WI, USA) the recovered luciferase activity was measured over $2 \mathrm{~s} /$ well in a Berthold Centro XS3 luminometer (Berthold Technologies, Bad Wildbad, Germany). Raw data were converted to arbitrary units (AU) using either a local positive serum as index or serial dilution of a SARS-CoV-2 spike protein antibody-positive serum. Thresholds for antibody positivity were established using a QQ plot analysis by selecting AU values at which the distribution of calculated AUs deviated from normality.

Statistical analysis Continuous variables are reported as median with IQR in parenthesis, time-related variables are reported as median and 95\% CI, while categorical variables are reported as frequency or per cent. Continuous variables were compared using the Wilcoxon rank sum or Kruskal-Wallis test; categorical variables were compared using the $\chi^{2}$ or Fisher's exact test. Imputation for missing data was not performed. The time-to-event was calculated from the date of symptoms onset to the date of the event, or the date of last visit, whichever occurred first. Survival rate was estimated according to Kaplan-Meier. To evaluate the association between patients' characteristics, laboratory findings and time to death or time to ICU admission, we used univariate and multivariate Cox proportional hazards models. The effect was reported as HR with the corresponding 95\% CI, estimated using the Wald approximation. All analysis of survival rate and associations were stratified according to time from symptoms to blood sampling ( $\leq 7$ days; $8-14$ days; $15-21$ days; $>21$ days) and antibody positivity was considered as a timevarying covariate.

The multivariate analysis was performed including variables significant at the level of $<0.1$ in the univariate analysis. Two-tailed $p$ values are reported, with a $p$ value $<0.05$ indicating statistical significance. All confidence intervals are two-sided and not adjusted for multiple testing. Statistical analyses were performed using SPSS 24 (SPSS/IBM, Armonk, NY, USA).

\section{Results}

Study participants From 25 February to 19 April 2020, 1031 consecutive adult cases with suspected COVID-19 infection were admitted to the Emergency or Clinical departments at the IRCCS San Raffaele Hospital (electronic supplementary material [ESM] Fig. 1). A serum sample for the purpose of the study was available in 582 of the 1031 patients. A confirmed infection (defined as a SARS-CoV-2-positive RTPCR test from a nasal/throat swab and/or signs, symptoms and radiological findings suggestive of COVID-19 pneumonia) was present in 509 cases out of $582(87.5 \%)$. Among these, a total of 452 patients $(88.8 \%$ ) were hospitalised and 79 were admitted to ICU. As of 25 May 2020, median follow-up time after symptoms onset was 59 (95\% CI 58,60) days. Ninetythree patients died during follow-up (18.3\%). The date of symptoms onset was available for 480 out of 509 patients.

Prevalence of diabetes and clinical profile in patients with COVID-19 Among patients with confirmed COVID-19 pneumonia, comorbid diabetes and newly diagnosed diabetes accounted for $17.7 \%(n=90)$ and $9.6 \%(n=49)$ of the patients, respectively. The characteristics of the study participants according to diabetes status are reported in ESM Tables 1 and 2 . The associations between baseline variables and diabetes, either comorbid or newly diagnosed, were assessed using logistic regression. Older age (OR 1.02 [95\% CI 1.01, 1.04]), higher BMI at diagnosis (OR 1.09 [1.04, 1.14]), cardiovascular comorbidities (OR 2.77 [1.59, 4.82]), hypertension (OR $3.60[2.37,5.46]$ ) and chronic kidney disease (OR 2.17 [1.22, 3.88]) were all associated with diabetes. As for the treatment of diabetes at the diagnosis of COVID-19 pneumonia, $35.3 \%$ of the patients were untreated (newly diagnosed diabetes), $7.9 \%$ were treated with lifestyle modifications, $36.7 \%$ with non-insulin oral or injectable glucose-lowering medications, $13.7 \%$ with insulin and $6.4 \%$ with insulin and oral diabetes medications. The median time from COVID-19 symptoms onset to hospital admission was 7 (4-9) and 7 (5-11) days for patients with and without diabetes, respectively. With the exception of a reduced prevalence of cough, patients with diabetes did not report different symptoms at the time of hospital admission than patients without diabetes (ESM Table 3). At the time of blood sampling for measuring the humoral response (median days: 10 [7-15.5] and 11 [7-16] in patients with or without diabetes, respectively, $p=0.136$ ), the presence of diabetes, either comorbid or newly diagnosed, was associated with worse lung $\left(\mathrm{PaO}_{2} /\right.$ $\mathrm{FiO}_{2}$ ratio [partial pressure of $\mathrm{O}_{2} /$ fraction of inspired $\mathrm{O}_{2}$ ]: 171 [103-286] vs 241 [120-342], $p=0.027$; see ESM Table 1) and kidney (serum creatinine: $94.6[75.8-134.6]$ vs 72.4 [58.7-93.8] $\mu \mathrm{mol} / \mathrm{l}, p=0.003$; see ESM Table 3) function. Furthermore, the presence of diabetes was associated with an increase in neutrophils $\backslash$ white blood cell count 
Table 1 Diabetes and COVID-19 clinical outcome

\begin{tabular}{|c|c|c|c|c|c|c|c|}
\hline \multirow[t]{2}{*}{ Characteristics } & \multirow[t]{2}{*}{ Discharged } & \multicolumn{2}{|c|}{ Hospitalised, ward } & \multicolumn{2}{|c|}{ Hospitalised, ICU } & \multirow[t]{2}{*}{ Dead } & \multirow[t]{2}{*}{$p$ value } \\
\hline & & $\leq 7$ days & $>7$ days & Alive & Dead & & \\
\hline$N$ & 57 & 78 & 232 & 49 & 30 & 63 & \\
\hline Age, years & $49(44-61)$ & $61(50-68)$ & $64(56-76)$ & $61(52-67)$ & $69(62-72)$ & $80(73-85)$ & $<0.001$ \\
\hline Male sex, $\%$ & 47 & 63 & 68 & 90 & 67 & 62 & $<0.001$ \\
\hline Diabetes, $\%$ & 15.8 & 12.8 & 25 & 32.6 & 60 & 44.5 & $<0.001$ \\
\hline Comorbid & 8.8 & 11.5 & 18.5 & 16.3 & 20 & 30.2 & \\
\hline Newly diagnosed & 7 & 1.3 & 6.5 & 16.3 & 40 & 14.3 & \\
\hline \multicolumn{8}{|l|}{ Diabetes, $\%$} \\
\hline Type 1 & 0 & 0 & 0 & 2 & 0 & 3.2 & $<0.001$ \\
\hline Type 2 & 16 & 11 & 24 & 31 & 60 & 35 & \\
\hline Metasteroid & 0 & 0 & 0.4 & 0 & 0 & 5 & \\
\hline Pancreatogenic & 0 & 1.3 & 0.4 & 0 & 0 & 1.6 & \\
\hline \multicolumn{8}{|l|}{ FPG, mmol/1 } \\
\hline Median & $5.5(5.1-6.2)$ & $5.2(4.8-5.7)$ & $5.4(4.8-6.2)$ & $6.3(5.3-7.9)$ & $7.7(6.6-11.4)$ & $6.3(5.3-9.1)$ & $<0.001$ \\
\hline Max & $5.6(5.1-6.6)$ & $5.8(5.2-6.6)$ & $6.3(5.5-7.7)$ & $7.1(6.1-10.2)$ & $9.5(8.1-11.9)$ & $6.9(5.8-11)$ & $<0.001$ \\
\hline Min & $5.3(4.9-5.9)$ & $4.7(4.2-5.1)$ & $4.6(4-5.4)$ & $5.2(4.4-6.4)$ & $5.9(5.1-7.2)$ & $5.4(4.2-6.9)$ & $<0.001$ \\
\hline SD & $0.9(0.6-1.2)$ & $0.7(0.4-1.2)$ & $0.8(0.5-1.4)$ & $1.2(0.7-2.5)$ & $1.8(1.1-2.7)$ & $1.5(0.2-2.9)$ & $<0.001$ \\
\hline No. of determinations & $1(1-1)$ & $2(2-3)$ & $3(2-7)$ & $3(2-6.5)$ & $2(2-4)$ & $2(2-3.5)$ & $<0.001$ \\
\hline
\end{tabular}

Data are presented as median (IQR) or percentage

Continuous variables between clinical outcome groups were compared using Kruskal-Wallis test; categorical variables were compared using the $\chi^{2}$ or Fisher's exact test

(neutrophils: $5.8[4.1-9.6]$ vs $4.8[3.35-7.6] \times 109 / 1, p=$ 0.001 ; white blood cells: 8.25 [5.6-11.7] vs 6.7 [5-9.3] $\times$ 109/1; $p<0.001$ ), inflammation (serum CRP: 82.4 [24.1157] vs 59.1 [19.5-131.9] $\mathrm{mg} / \mathrm{l}, p=0.026)$, tissue damage (serum LDH: 6.66 [5.29-8.93] vs 5.9 [4.49-7.62] $\mu \mathrm{kat} / 1$, $p=0.003$ ) and coagulatory cascade activation markers (Ddimer: 10.8 [5.37-19.5] vs 4.82 [2.41-9.64] $\mathrm{nmol} / \mathrm{l}$, $p<0.001)$, as well as a decrease in haemoglobin $(125$ [111.5-140] vs 132 [118-144] g/l, $p=0.011$ ) (see ESM Table 3).

\section{Effect of diabetes and hyperglycaemia on COVID-19 clinical} outcome Patients were classified in six groups based on COVID-19 pneumonia severity: (1) discharged without hospitalisation (57 out of 509, 11.2\%); hospitalised and alive: (2) $\leq 7$ days ( 78 out of $509,15.3 \%$ ); (3) $>7$ days (232 out of 509, 45.6\%); hospitalised and admitted to ICU: (4) alive (49 out of $509,9.6 \%$ ); (5) deceased (30 out of 509, 5.9\%); (6) hospitalised and deceased on a hospital ward (63 out of 509, $12.4 \%)$. None of the patients eventually discharged died during follow-up. Going from the most favourable to the worst clinical outcome, the prevalence of diabetes, either comorbid or newly diagnosed, increased $(15.8 \%, 12.8 \%$, $25.0 \%, 32.7 \%, 60.0 \%, 44.4 \%$, respectively; $p<0.001)$. Accordingly, mean FPG and glucose variability were higher in patients with more severe disease (Table 1). Results of univariate and multivariate survival rate analysis according to diabetes status and FPG levels are reported in ESM Table 4 and Fig. 1a-f. Multivariate analysis showed that diabetes status (either comorbid or newly diagnosed) (HR $2.32[95 \%$ CI 1.44, 3.75] $p=0.001)$, mean FPG $($ HR $1.14 \times$ $1.1 \mathrm{mmol} / 1[95 \%$ CI $1.08,1.21] p<0.001)$ and glucose variability (HR $1.08 \times 0.6 \mathrm{mmol} / 1$ [95\% CI 1.03, 1.15] $p=0.002)$ were independently associated with an increased risk of mortality. Concordantly, these three variables were also associated with an increased risk of ICU admission (ESM Table 5). To evaluate the specific impact of glucose levels and glucose variability, the same analysis was conducted separately for patients with and without diabetes. In participants with diabetes, both mean FPG $(\mathrm{HR} 1.13 \times 1.1 \mathrm{mmol} / 1$ [95\% CI 1.01, 1.26], $p=0.026)$ and glucose variability (HR $1.13 \times 0.6 \mathrm{mmol} / 1$ [95\% CI 1.03, 1.24], $p=0.009$ ) were independently associated with an increased risk of death (ESM Table 4). Moreover, in patients with comorbid diabetes, treatment intensity (lifestyle changes < oral diabetes medications or insulin associated with oral diabetes medications $<$ insulin alone) was associated with an increased risk of death (Fig. 1c). Unexpectedly, even in patients without diabetes, we found an association between mean FPG and mortality risk (HR $2.39 \times$ $1.1 \mathrm{mmol} / 1$ [95\% CI 1.49, 3.81], $p<0.001$; ESM Table 4) or risk of ICU admission (HR $2.49 \times 1.1 \mathrm{mmol} / \mathrm{l}$ [95\% CI 1.5, 4.12], $p<0.001$; ESM Table 5). 

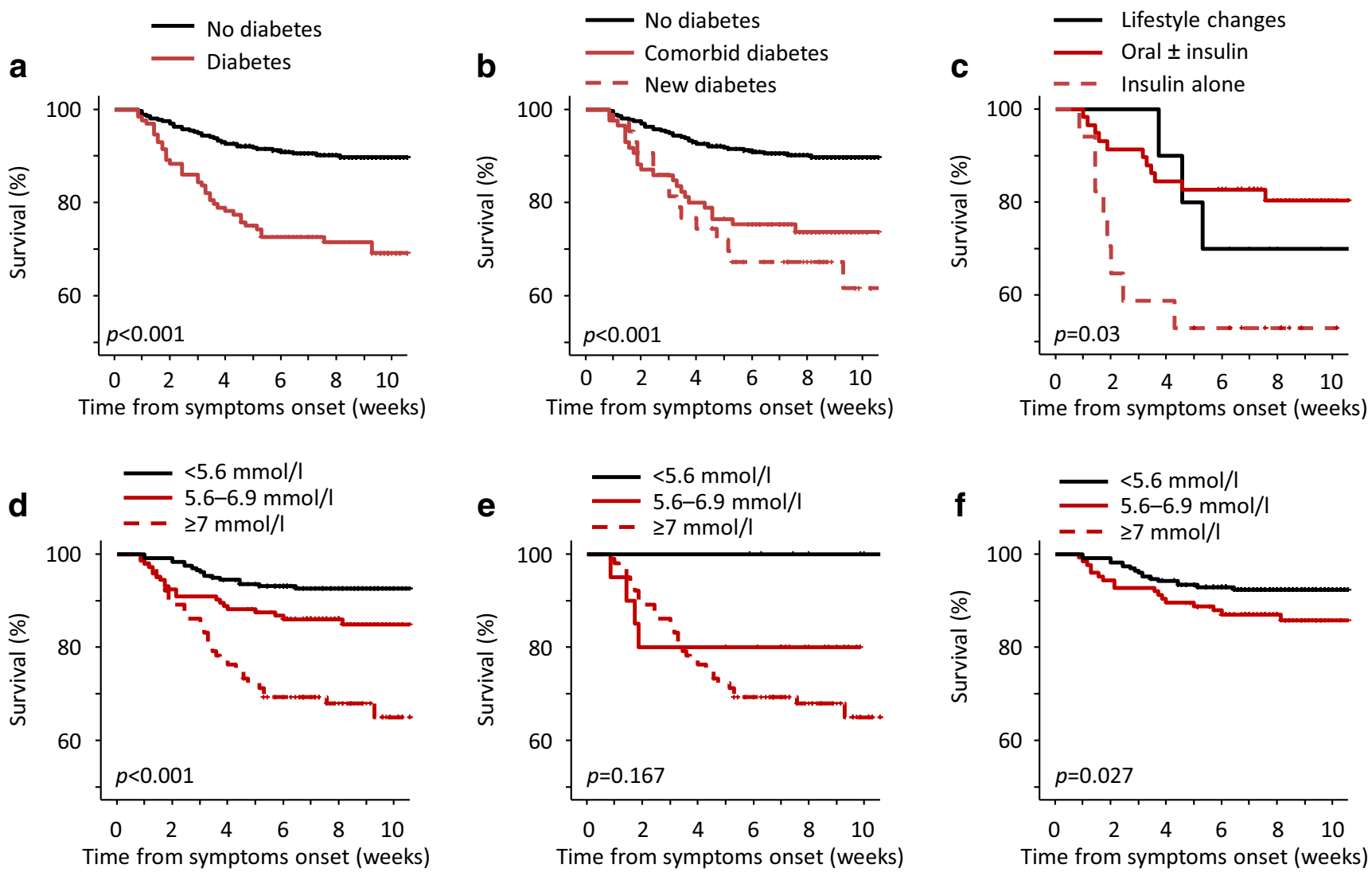

Fig. 1 Kaplan-Meier estimated survival rate of patients with COVID-19 pneumonia. Among 480 participants for whom COVID-19 symptoms onset date was available, survival rate was estimated by: (a) presence of diabetes (diabetes $n=128$; no diabetes $n=352$ ); (b) comorbid or newly diagnosed diabetes ( $n=85$ and $n=43$, respectively); and (c) treatment intensity in patients with comorbid diabetes (lifestyle changes $n=10$; oral glucose-lowering agents with or without insulin $n=58$; insulin alone $n=$

\section{Antibody responses to SARS-CoV-2 stratified by diabetes or} glucose levels The results of the extended analysis of the IgG, $\mathrm{IgM}$ and $\mathrm{IgA}$ responses to the SARS-CoV-2 spike protein (RBD or $\mathrm{S} 1+\mathrm{S} 2$ ) and $\mathrm{IgG}$ to NP in our cohort, stratified according to the presence of diabetes or FPG are reported in Fig. 2 (antibody prevalence) and ESM Fig. 2 (antibody titres). In patients with COVID-19 pneumonia, the anti-RBD IgG, the anti-S1+S2 IgG and the anti-NP IgG titres increased from week 1 to week 3, followed by stabilisation or marginal decline at week 4 and beyond (ESM Fig. 2). Similarly, the IgM titre of anti-RBD and anti-S1+S2 antibodies increased from week 1 to week 2 or 3 , followed by a decline thereafter (ESM Fig. 2). Concordantly, we observed an increase in all antibody prevalence with the duration of symptoms (Fig. 2a, b). Marginal differences between patients with and without diabetes were evident, including a higher quantitative response of anti-RBD IgG at week 2 and 3 after symptoms onset and an earlier decline of $\operatorname{IgM}$ titre at week 3 in patients with diabetes compared with those without diabetes (ESM Fig. 2 and ESM Tables 6 and 7). Anti-RBD and S1+S2 antibodies of the IgA class showed higher titres and prevalence
17). In addition, survival rate was evaluated by median FPG (d) ( $<5.6 \mathrm{mmol} / \mathrm{l} n=235 ; 5.6-6.9 \mathrm{mmol} / 1 \mathrm{l} n=144 ; \geq 7 \mathrm{mmol} / 1 \mathrm{l} n=101)$, and separately in patients with diabetes $(\mathbf{e})(<5.6 \mathrm{mmol} / 1 \mathrm{n}=7 ; 5.6-6.9 \mathrm{mmol} / \mathrm{l}$ $n=20 ; \geq 7 \mathrm{mmol} / 1 n=101)$ or without diabetes (f) $(<5.6 \mathrm{mmol} / 1 n=228$; $5.6-6.9 \mathrm{mmol} / \mathrm{l} n=124 ; \geq 7 \mathrm{mmol} / \mathrm{l} n=0)$. The logrank test was used to test differences in estimated survival rate. Crosses indicate censored patients (censoring for lack of follow-up data)

according to the duration of symptoms regardless of the presence of diabetes.

Antibody responses to SARS-CoV-2 and clinical outcome in participants with and without diabetes We performed a time-dependent multivariate Cox regression analysis of antibody responses and time to death, adjusted for sex, age and presence of diabetes, and stratified for symptom duration at the time of sampling (Table 2). The development of SARSCoV-2 RBD IgG antibodies was associated with improved patient survival rate in regression analysis with an $\mathrm{HR}$ for time to death of $0.4(95 \% \mathrm{CI} 0.23,0.71, p=0.002)$. The protective effect of RBD IgG positivity was confirmed, even when the analysis was performed separately in patients with (HR 0.37 [95\%CI $0.17,0.81], p=0.013$ ) or without diabetes (HR 0.43 [95\% CI 0.19, 0.95], $p=0.038)$. A less consistent effect on survival rate was also present for the development of $\mathrm{IgG}$ to SARS-CoV-2 S1+S2 (whole population: HR 0.53 [95\% CI $0.31,0.90$ ], $p=0.018$; with diabetes HR 0.47 [95\% CI 0.21 , 1.01], $p=0.052$; without diabetes: HR 0.62 [95\% CI 0.29 , 1.32], $p=0.619)$. Neither the response to NP nor the response 

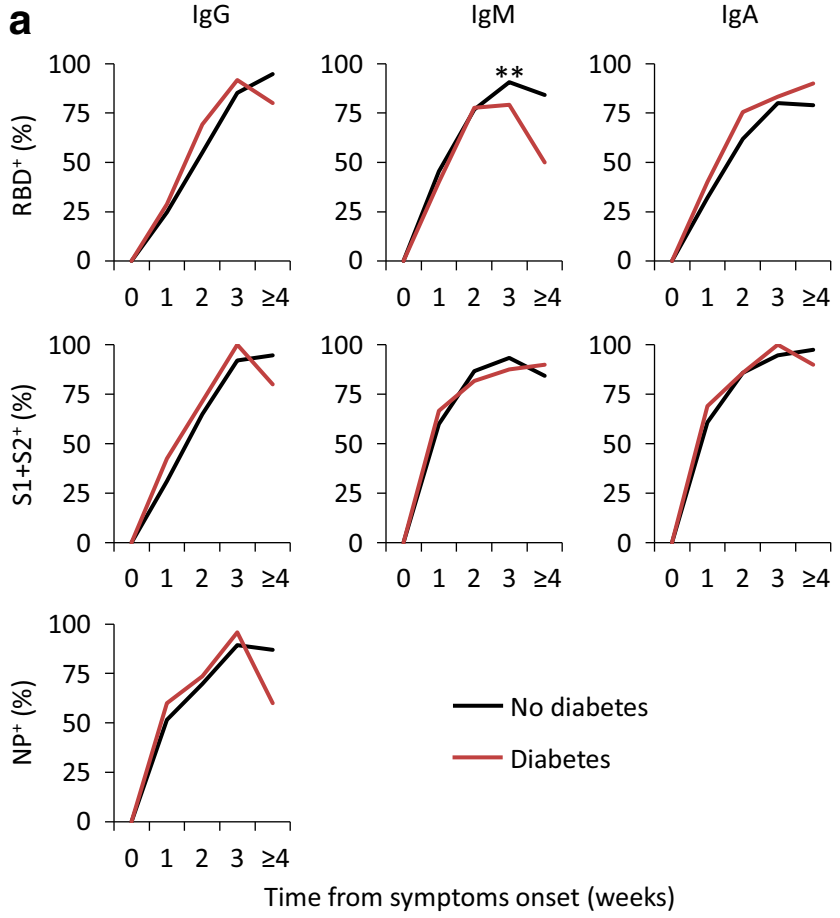

Fig. 2 Antibody prevalence based on diabetes and median FPG. Kinetics of anti-SARS-CoV-2 RBD, S1+S2 and NP IgG, IgA and IgM antibody development, stratified by diabetes status (a) or mean glucose levels (b) in patients with COVID-19 pneumonia. For each time point, the results are

to the same antigens of other immunoglobulin classes were associated with patient survival rate.

\section{Discussion}

To date, the pathophysiological and virologic mechanisms underpinning the strong association between diabetes and risk of severe/critical illness or increased in-hospital mortality risk in patients with COVID-19 pneumonia are poorly elucidated. In our study, we evaluated whether diabetes affects the ability to mount an appropriate humoral response against SARS$\mathrm{CoV}-2$. Since people with diabetes are at high risk for the severe forms of COVID-19 pneumonia, they are likely to be among the first to benefit from a future vaccination against SARS-CoV-2. Therefore, knowing whether the humoral response against SARS-CoV-2 in individuals with diabetes is present and superimposable to that of those without diabetes is of fundamental importance. For this study, we used a LIPS liquid phase immunoassay developed on the basis of our prior expertise. The description of the assay performance and potential caveats $[27,28]$ falls outside the scope of this study and is being published elsewhere [25]. Taken together, our data support the evidence of an efficient humoral response in patients with diabetes, as the prevalence of positivity for the different classes of antibodies to multiple SARS-CoV-2 antigens was superimposable, as for timing and antibody titres, to
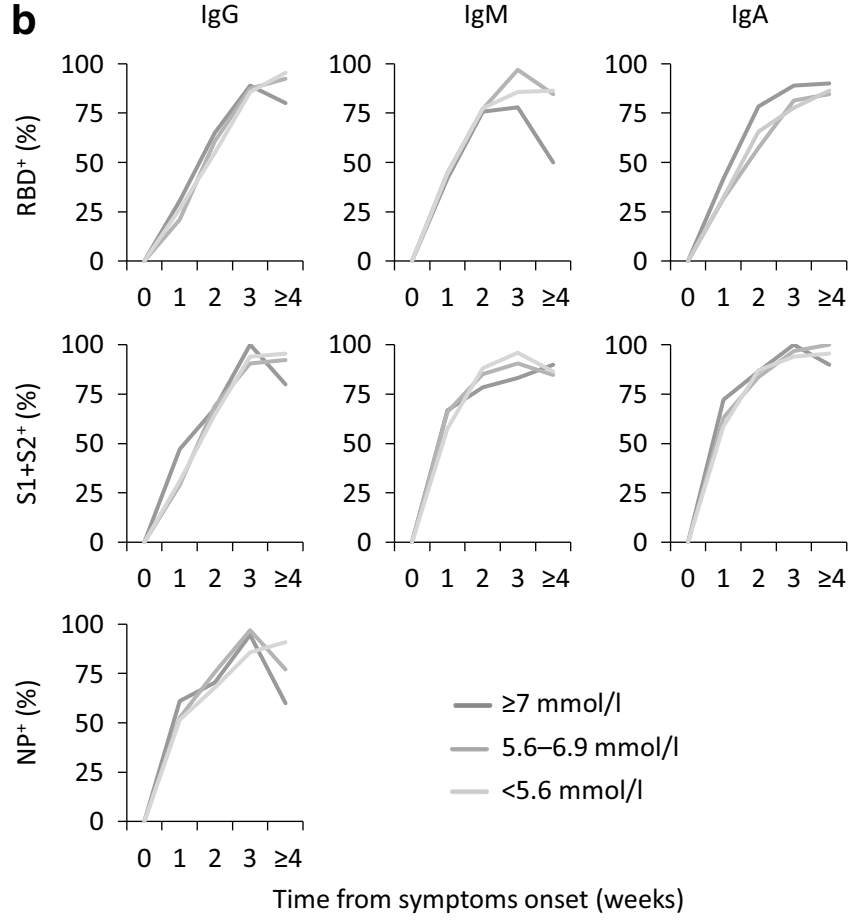

shown as median of percentage of positivity (week 1: no diabetes $n=97$, diabetes $n=45$; week 2 : no diabetes $n=142$, diabetes $n=49$; week 3 : no diabetes $n=75$, diabetes $n=24$; week $\geq 4$ : no diabetes $n=38$, diabetes $n=10)$. ${ }^{* *} p<0.01$ by $\chi^{2}$ test, diabetes vs no diabetes

that of non-diabetic patients and was not influenced by glucose levels. A higher quantitative response of anti-RBD $\operatorname{IgG}$ at weeks 2 and 3 after the onset of symptoms and an earlier decline of IgM titre at week 3 was present in individuals with diabetes compared with those without (ESM Fig. 2 and ESM Table 6 and 7). These marginal differences might be a random effect or could represent a specific characteristic of the humoral response of people with diabetes. As for the second hypothesis, we can speculate that the presence of a more pronounced inflammatory state in patients with diabetes (as supported by higher values of neutrophil count and CRP) might favour an early maturation of the antibody response. Moreover, both obese individuals and those with type 2 diabetes have a decreased percentage of the anti-inflammatory B cell subset and an increased percentage of proinflammatory memory B cells [29], and this might have contributed to the observed differences.

More generally, whether hyperglycaemia modulates the antibody response to a virus is still a matter of discussion [30]. Several defects in immunity have been associated with hyperglycaemia/insulin resistance, including inhibited lymphocyte proliferative response, impaired monocyte/ macrophage and neutrophil function, abnormal delayed type hypersensitivity reaction and complement activation dysfunction [31]. Individuals with diabetes have a consistently decreased immunological response to the hepatitis $B$ vaccine [32], while less consistent results were noted for influenza and 
Table 2 Multivariable covariate Cox regression analysis of antibody responses on the time to death

\begin{tabular}{|c|c|c|c|c|c|c|}
\hline \multirow[t]{2}{*}{ Characteristics } & \multicolumn{2}{|l|}{ All $(n=480)$} & \multicolumn{2}{|c|}{$\begin{array}{l}\text { Diabetes (either comorbid or } \\
\text { newly diagnosed) }(n=128)\end{array}$} & \multicolumn{2}{|c|}{ No diabetes $(n=352)$} \\
\hline & $\mathrm{HR}(95 \% \mathrm{CI})$ & $p$ value & $\operatorname{HR}(95 \% \mathrm{CI})$ & $p$ value & $\mathrm{HR}(95 \% \mathrm{CI})$ & $p$ value \\
\hline Age, years & $1.06(1.04,1.08)$ & $<0.001$ & $1.05(1.02,1.08)$ & 0.002 & $1.07(1.04,1.09)$ & $<0.001$ \\
\hline Male sex & $1.3(0.80,2.12)$ & 0.292 & $1.25(0.61,2.58)$ & 0.54 & $1.34(0.68,2.62)$ & 0.394 \\
\hline Diabetes & $3.00(1.87,4.81)$ & $<0.001$ & - & - & - & - \\
\hline IgG RBD & $0.40(0.23,0.71)$ & 0.002 & $0.37(0.17,0.81)$ & 0.013 & $0.43(0.19,0.95)$ & 0.038 \\
\hline Age, years & $1.06(1.04,1.08)$ & $<0.001$ & $1.05(1.02,1.09)$ & 0.001 & $1.07(1.04,1.09)$ & $<0.001$ \\
\hline Male sex & $1.26(0.77,2.05)$ & 0.353 & $1.28(0.62,2.63)$ & 0.503 & $1.33(0.67,2.63)$ & 0.407 \\
\hline Diabetes & $2.77(1.73,4.43)$ & $<0.001$ & - & - & - & - \\
\hline IgM RBD & $0.76(0.46,1.24)$ & 0.275 & $0.87(0.43,1.76)$ & 0.705 & $0.61(0.30,1.25)$ & 0.182 \\
\hline Age, years & $1.06(1.04,1.08)$ & $<0.001$ & $1.05(1.02,1.09)$ & 0.001 & $1.06(1.04,1.09)$ & $<0.001$ \\
\hline Male sex & $1.23(0.75,2.00)$ & 0.406 & $1.29(0.63,2.65)$ & 0.491 & $1.2(0.61,2.33)$ & 0.597 \\
\hline Diabetes & $2.86(1.78,4.58)$ & $<0.001$ & - & - & - & - \\
\hline IgA RBD & $0.81(0.49,1.34)$ & 0.414 & $0.99(0.49,2.02)$ & 0.982 & $0.67(0.33,1.38)$ & 0.277 \\
\hline Age, years & $1.06(1.04,1.08)$ & $<0.001$ & $1.05(1.02,1.08)$ & 0.001 & $1.07(1.04,1.09)$ & $<0.001$ \\
\hline Male sex & $1.23(0.76,2.01)$ & 0.399 & $1.21(0.58,2.48)$ & 0.612 & $1.25(0.64,2.45)$ & 0.505 \\
\hline Diabetes & $2.98(1.86,4.78)$ & $<0.001$ & - & - & - & - \\
\hline IgG S1+S2 & $0.53(0.31,0.90)$ & 0.018 & $0.47(0.21,1.01)$ & 0.052 & $0.62(0.29,1.32)$ & 0.619 \\
\hline Age, years & $1.07(1.04,1.09)$ & $<0.001$ & $1.06(1.02,1.09)$ & $<0.001$ & $1.07(1.04,1.1)$ & $<0.001$ \\
\hline Male sex & $1.30(0.80,2.12)$ & 0.295 & $1.27(0.62,2.60)$ & 0.521 & $1.38(0.69,2.76)$ & 0.369 \\
\hline Diabetes & $2.86(1.79,4.58)$ & $<0.001$ & - & - & - & - \\
\hline IgM S1+S2 & $0.60(0.35,1.03)$ & 0.065 & $0.61(0.29,1.3)$ & 0.206 & $0.59(0.26,1.33)$ & 0.202 \\
\hline Age, years & $1.06(1.04,1.08)$ & $<0.001$ & $1.05(1.02,1.09)$ & 0.001 & $1.07(1.04,1.1)$ & $<0.001$ \\
\hline Male sex & $1.23(0.75,2.00)$ & 0.409 & $1.27(0.62,2.63)$ & 0.511 & $1.23(0.63,2.39)$ & 0.548 \\
\hline Diabetes & $2.83(1.76,4.52)$ & $<0.001$ & - & - & - & - \\
\hline $\mathrm{IgA} \mathrm{S} 1+\mathrm{S} 2$ & $0.77(0.44,1.34)$ & 0.357 & $0.90(0.41,1.99)$ & 0.802 & $0.70(0.32,1.53)$ & 0.375 \\
\hline Age, years & $1.06(1.04,1.09)$ & $<0.001$ & $1.05(1.02,1.09)$ & 0.001 & $1.07(1.04,1.1)$ & $<0.001$ \\
\hline Male sex & $1.24(0.76,2.02)$ & 0.386 & $1.27(0.62,2.61)$ & 0.514 & $1.27(0.65,2.47)$ & 0.488 \\
\hline Diabetes & $2.85(1.78,4.56)$ & $<0.001$ & - & - & - & - \\
\hline IgG NP & $0.67(0.40,1.10)$ & 0.116 & $0.77(0.37,1.61)$ & 0.492 & $0.61(0.30,1.24)$ & 0.174 \\
\hline
\end{tabular}

varicella zoster vaccines [33, 34]. The immunological response to a future SARs-CoV-2 vaccine will be assessed when the vaccine becomes available; however, our data allow a cautious optimism regarding effective immunisation in individuals with diabetes, as well as in the general population. Together with the evidence that the antibody response against SARS-CoV-2 antigens in patients with diabetes is superimposable, with only marginal differences, to that of nondiabetic patients, our data also suggest that the positivity for IgG against the SARS-CoV-2 spike RBD, one of the more relevant targets of vaccine strategies [35], is predictive of survival rate, both in the presence or absence of diabetes (see Table 2). This finding was not obvious. In fact, a similar antibody response but a worse outcome might suggest that the humoral response is not protective in individuals with diabetes. However, our analysis of the humoral response vs clinical outcome does not support this hypothesis (see Table 2), confirming the relevance for patient survival rate of the specific antigen response against spike RBD even in the presence of diabetes, and it underlines how the mechanism explaining the worse clinical outcome in patients with diabetes is unrelated to the antibody response. This, together with evidence that increased blood glucose levels do predict a poor prognosis even in non-diabetic individuals and the association with increased levels of inflammatory biomarkers and hypercoagulopathy, as well as leucocytosis and neutrophilia, support the speculation that glucose per se could be an independent biological negative factor, acting as a direct regulator of innate immunity [36].

In addition to the humoral response data, our cohort also provided additional insights into the relationship between diabetes, hyperglycaemia and COVID-19 pneumonia. First, we confirmed that hyperglycaemia was independently associated with a poorer in-hospital outcome, even after the results 
were adjusted for age, sex, obesity and other relevant comorbidities, as recently reported [37]. Second, patients with a diagnosis of diabetes, either comorbid or newly diagnosed, with better controlled blood glucose levels had less severe COVID-19 pneumonia and lower mortality risk compared with those with poorly controlled blood glucose during hospitalisation [38]. This suggests that improving glucose control could be a critical measure to improve outcomes in patients with COVID-19 pneumonia and strongly supports the need for an algorithm for hyperglycaemia treatment, validated through a randomised clinical trial [39]. Third, intensity of diabetes treatment in patients with comorbid diabetes was associated with higher mortality risk. Fourth, glucose levels were associated with the severity of COVID-19 pneumonia and mortality risk even in individuals without diabetes, confirming the result of a retrospective study cohort recently reported by two hospitals based in Wuhan, China [40]. Finally, patients with newly diagnosed diabetes had an increased severity of COVID-19 pneumonia and higher mortality risk. The classification of COVID-19 patients with newly diagnosed diabetes is difficult [41] and there are no previous studies clearly assessing these individuals. In our cohort individuals with newly diagnosed diabetes represented one third of those with diabetes. They may be individuals with a previously unrecognised diabetes, or with stress hyperglycaemia or with a new onset diabetes associated with COVID-19 pneumonia. In our cohort, they were similar in age and comorbidity burden to patients with comorbid diabetes. Moreover, levels of inflammatory biomarkers, hypercoagulopathy, leucocytosis and neutrophilia were also comparable to those seen in patients with comorbid diabetes. However, we did not have access to the medical records prior to this hospitalisation and, therefore, we were unable to determine the role of prior glycaemic status.

A major strength of this study is that it was conducted in a large cohort of non-Asian individuals, as previous studies were mainly performed in patients of Asian ancestry, mostly Chinese [42]. Furthermore, the cohort was well characterised with regard to comorbidities, allowing adjustment of our results for age, sex, obesity and other factors. However, this study encompasses some limitations. First, even if we detected a rise in antibodies against SARS-CoV-2 after infection in patients with diabetes, we know little about their efficacy in clearing the virus. Moreover, the use of a longitudinal rather than a cross-sectional design would improve future studies. Second, while there is substantial evidence of a strong association between hyperglycaemia and poorer prognosis, the observational design of our study did not allow us to prove causality. Third, we were not able to provide detailed information on $\mathrm{HbA}_{1 \mathrm{c}}$ levels in our cohort. Finally, our cohort is limited to hospitalised patients and we should be cautious in generalising our findings to people with SARs-CoV-2 infection with few or no symptoms.
In conclusion, patients with either diagnosed or undiagnosed diabetes are more likely to experience severe symptoms and death from COVID-19 pneumonia. Frailty, pre-existing comorbid conditions and a potential underlying immune system dysfunction could contribute to a poorer outcome. We documented that hyperglycaemia does not impair the humoral immune response against SARs-CoV-2. The evidence that RBD IgG positivity is associated with a remarkable protective effect (about $60 \%$ reduction) in patients with diabetes allows for a cautious optimism on the efficacy of future vaccines against SARs-COV-2 in people with diabetes. On the other hand, blood glucose levels were strongly associated with an increased mortality risk for COVID-19 pneumonia. It is not possible to prove causality on the basis of the available observational studies, and further longitudinal studies are needed to draw definitive conclusions.

Data availability No data outside those published in this manuscript and the material presented in the ESM are available for sharing.

Funding This research received no specific grant from any funding agency in the public, commercial or not-for-profit sectors.

Authors' relationships and activities The authors declare that there are no relationships or activities that might bias, or be perceived to bias, their work.

Contribution statement LP and VL contributed to the conception of the study, wrote the manuscript, researched data and contributed to the discussion. MSe, EBa, $\mathrm{CB}$ and IM contributed to the acquisition and analysis of antibody data and revised the manuscript. AM, RM, CT and FC contributed to the acquisition of samples, managed the biobanking activities and critically revised the manuscript, recruited patients and managed sample biobanking. AD, AC, AL SM, CM, LDF and GV contributed to the acquisition, analysis and interpretation of clinical data and critically revised the manuscript. PRQ researched data, and designed and managed data collection and eCRFs and critically revised the manuscript. EBo, GL and MSc contributed to the design of the study and critically reviewed/edited the manuscript. LP is the guarantor of this work and, as such, had full access to all the data presented in the study and takes responsibility for the integrity of the data and the accuracy of the data analysis. The final manuscript has been read and approved by all named authors.

\section{References}

1. Zhu N, Zhang D, Wang W et al (2020) A novel coronavirus from patients with pneumonia in China, 2019. N Engl J Med 382(8): 727-733. https://doi.org/10.1056/NEJMoa2001017

2. Xu L, Mao Y, Chen G (2020) Risk factors for 2019 novel coronavirus disease (COVID-19) patients progressing to critical illness: a systematic review and meta-analysis. Aging (Albany NY) 12(12): 12410-12421. https://doi.org/10.18632/aging.103383

3. Wang D, Hu B, Hu C et al (2020) Clinical characteristics of 138 hospitalized patients with 2019 novel coronavirus-infected pneumonia in Wuhan, China. JAMA 323(11):1061-1069. https://doi. org/10.1001/jama.2020.1585

4. Wu C, Chen X, Cai Y et al (2020) Risk factors associated with acute respiratory distress syndrome and death in patients with coronavirus 
disease 2019 pneumonia in Wuhan, China. JAMA Intern Med 180(7):934-943. https://doi.org/10.1001/jamainternmed.2020. 0994

5. Huang C, Wang Y, Li X et al (2020) Clinical features of patients infected with 2019 novel coronavirus in Wuhan, China. Lancet 395(10223):497-506. https://doi.org/10.1016/S0140-6736(20) 30183-5

6. Liu W, Tao ZW, Wang L et al (2020) Analysis of factors associated with disease outcomes in hospitalized patients with 2019 novel coronavirus disease. Chin Med J 133(9):1032-1038. https://doi. org/10.1097/CM9.0000000000000775

7. Guan WJ, Ni ZY, Hu Y et al (2020) Clinical characteristics of coronavirus disease 2019 in China. N Engl J Med 382(18):17081720. https://doi.org/10.1056/NEJMoa2002032

8. Mantovani A, Byrne CD, Zheng MH, Targher G (2020) Diabetes as a risk factor for greater COVID-19 severity and in-hospital death: a meta-analysis of observational studies. Nutr Metab Cardiovasc Dis 30(8):1236-1248. https://doi.org/10.1016/j.numecd.2020.05.014

9. Yang JK, Feng Y, Yuan MY et al (2006) Plasma glucose levels and diabetes are independent predictors for mortality and morbidity in patients with SARS. Diabet Med 23(6):623-628. https://doi.org/10. 1111/j.1464-5491.2006.01861.x

10. Kulcsar KA, Coleman CM, Beck SE, Frieman MB (2019) Comorbid diabetes results in immune dysregulation and enhanced disease severity following MERS-CoV infection. JCI Insight 4(20): e131774. https://doi.org/10.1172/jci.insight.131774

11. Banik GR, Alqahtani AS, Booy R, Rashid H (2016) Risk factors for severity and mortality in patients with MERS-CoV: analysis of publicly available data from Saudi Arabia. Virol Sin 31(1):81-84. https://doi.org/10.1007/s12250-015-3679-z

12. Apicella M, Campopiano MC, Mantuano M, Mazoni L, Coppelli A, Del Prato S (2020) COVID-19 in people with diabetes: understanding the reasons for worse outcomes. Lancet Diabetes Endocrinol 8(9):782-792. https://doi.org/10.1016/S2213-8587(20) 30238-2

13. Rao S, Lau A, So HC (2020) Exploring diseases/traits and blood proteins causally related to expression of ACE2, the putative receptor of SARS-CoV-2: a Mendelian randomization analysis highlights tentative relevance of diabetes-related traits. Diabetes Care 43(7):1416-1426. https://doi.org/10.2337/dc20-0643

14. Fernandez C, Rysa J, Almgren P et al (2018) Plasma levels of the proprotein convertase furin and incidence of diabetes and mortality. J Intern Med 284(4):377-387. https://doi.org/10.1111/joim.12783

15. Ji HL, Zhao R, Matalon S, Matthay MA (2020) Elevated plasmin(ogen) as a common risk factor for COVID-19 susceptibility. Physiol Rev 100(3):1065-1075. https://doi.org/10.1152/physrev. 00013.2020

16. Wysocki J, Ye M, Soler MJ et al (2006) ACE and ACE2 activity in diabetic mice. Diabetes 55(7):2132-2139. https://doi.org/10.2337/ db06-0033

17. Ferrario CM, Jessup J, Chappell MC et al (2005) Effect of angiotensin-converting enzyme inhibition and angiotensin II receptor blockers on cardiac angiotensin-converting enzyme 2 . Circulation 111(20):2605-2610. https://doi.org/10.1161/ CIRCULATIONAHA.104.510461

18. Romani-Perez M, Outeirino-Iglesias V, Moya CM et al (2015) Activation of the GLP-1 receptor by liraglutide increases ACE2 expression, reversing right ventricle hypertrophy, and improving the production of SP-A and SP-B in the lungs of type 1 diabetes rats. Endocrinology 156(10):3559-3569. https://doi.org/10.1210/ en.2014-1685

19. Tikoo K, Patel G, Kumar S et al (2015) Tissue specific up regulation of ACE2 in rabbit model of atherosclerosis by atorvastatin: role of epigenetic histone modifications. Biochem Pharmacol 93(3): 343-351. https://doi.org/10.1016/j.bcp.2014.11.013
20. Chen X, Hu W, Ling J et al (2020) Hypertension and diabetes delay the viral clearance in COVID-19 patients. medRxiv: https://doi.org/ 10.1101/2020.03.22.20040774 (preprint). Version 24 March 2020

21. Guo W, Li M, Dong Y et al (2020) Diabetes is a risk factor for the progression and prognosis of COVID-19. Diabetes Metab Res Rev e3319. https://doi.org/10.1002/dmrr.3319

22. Geerlings SE, Hoepelman AI (1999) Immune dysfunction in patients with diabetes mellitus (DM). FEMS Immunol Med Microbiol 26(3-4):259-265. https://doi.org/10.1111/j.1574-695X. 1999.tb01397.x

23. Ciceri F, Castagna A, Rovere-Querini P et al (2020) Early predictors of clinical outcomes of COVID-19 outbreak in Milan, Italy. Clin Immunol 217:108509. https://doi.org/10.1016/j.clim.2020. 108509

24. Zangrillo A, Beretta L, Silvani P et al (2020) Fast reshaping of intensive care unit facilities in a large metropolitan hospital in Milan, Italy: facing the COVID-19 pandemic emergency. Crit Care Resusc 22(2):91-94

25. Secchi M, Bazzigaluppi E, Brigatti C et al (2020) COVID-19 survival associates with the immunoglobulin response to the SARS-CoV-2 spike receptor binding domain. J Clin Invest. https://doi.org/10.1172/JCI142804

26. Williams AJ, Norcross AJ, Chandler KA, Bingley PJ (2006) Nonspecific binding to protein A Sepharose and protein G Sepharose in insulin autoantibody assays may be reduced by pre-treatment with glycine or ethanolamine. J Immunol Methods 314(1-2):170-173. https://doi.org/10.1016/j.jim.2006.06.003

27. Whitman JD, Hiatt J, Mowery CT et al (2020) Test performance evaluation of SARS-CoV-2 serological assays. medRxiv. https:// doi.org/10.1101/2020.04.25.20074856 (preprint). Version 17 May 2020

28. Wang Q, Du Q, Guo B et al (2020) A method to prevent SARSCoV-2 IgM false positives in gold immunochromatography and enzyme-linked immunosorbent assays. J Clin Microbiol 58(6): e00375-e00320. https://doi.org/10.1128/JCM.00375-20

29. DeFuria J, Belkina AC, Jagannathan-Bogdan M et al (2013) B cells promote inflammation in obesity and type 2 diabetes through regulation of T-cell function and an inflammatory cytokine profile. Proc Natl Acad Sci U S A 110(13):5133-5138. https://doi.org/10.1073/ pnas. 1215840110

30. Zhai X, Qian G, Wang Y et al (2016) Elevated B cell activation is associated with type 2 diabetes development in obese subjects. Cell Physiol Biochem 38(3):1257-1266. https://doi.org/10.1159/ 000443073

31. Daryabor G, Atashzar MR, Kabelitz D, Meri S, Kalantar K (2020) The effects of type 2 diabetes mellitus on organ metabolism and the immune system. Front Immunol 11:1582. https://doi.org/10.3389/ fimmu.2020.01582

32. Schillie SF, Spradling PR, Murphy TV (2012) Immune response of hepatitis B vaccine among persons with diabetes: a systematic review of the literature. Diabetes Care 35(12):2690-2697. https:// doi.org/10.2337/dc12-0312

33. Dos Santos G, Tahrat H, Bekkat-Berkani R (2018) Immunogenicity, safety, and effectiveness of seasonal influenza vaccination in patients with diabetes mellitus: a systematic review. Hum Vaccin Immunother 14(8):1853-1866. https://doi.org/10. 1080/21645515.2018.1446719

34. Verstraeten T, Fletcher MA, Suaya JA et al (2020) Diabetes mellitus as a vaccine-effect modifier: a review. Expert Rev Vaccines 19(5):445-453. https://doi.org/10.1080/14760584.2020. 1760098

35. Rego GNA, Nucci MP, Alves AH et al (2020) Current clinical trials protocols and the global effort for immunization against SARSCoV-2. Vaccines (Basel) 8(3):E474. https://doi.org/10.3390/ vaccines 8030474 
36. Wang Q, Fang P, He R et al (2020) $O$-GlcNAc transferase promotes influenza A virus-induced cytokine storm by targeting interferon regulatory factor-5. Sci Adv 6(16):eaaz7086. https://doi.org/10. 1126/sciadv.aaz7086

37. Holman N, Knighton P, Kar P et al (2020) Risk factors for COVID19-related mortality in people with type 1 and type 2 diabetes in England: a population-based cohort study. Lancet Diabetes Endocrinol. https://doi.org/10.1016/S2213-8587(20)30271-0

38. Zhu L, She ZG, Cheng X et al (2020) Association of blood glucose control and outcomes in patients with COVID-19 and pre-existing type 2 diabetes. Cell Metab 31(6):1068-1077. https://doi.org/10. 1016/j.cmet.2020.04.021

39. Gianchandani R, Esfandiari NH, Ang L et al (2020) Managing hyperglycemia in the COVID-19 inflammatory storm. Diabetes. https://doi.org/10.2337/dbi20-0022
40. Wang S, Ma P, Zhang S et al (2020) Fasting blood glucose at admission is an independent predictor for 28-day mortality in patients with COVID-19 without previous diagnosis of diabetes: a multi-centre retrospective study. Diabetologia 63(10):2102-2111. https://doi.org/10.1007/s00125-020-05209-1

41. Rubino F, Amiel SA, Zimmet P et al (2020) New-onset diabetes in Covid-19. N Engl J Med 383(8):789-790. https://doi.org/10.1056/ NEJMc2018688

42. Abdi A, Jalilian M, Sarbarzeh PA, Vlaisavljevic Z (2020) Diabetes and COVID-19: a systematic review on the current evidences. Diabetes Res Clin Pract 166:108347. https://doi.org/10.1016/j. diabres.2020.108347

Publisher's note Springer Nature remains neutral with regard to jurisdictional claims in published maps and institutional affiliations. 